\title{
3D Reconstruction of a Spinal Motion Segment from 2D Medical Images: Objective Quantification of the Geometric Accuracy of the FE Mesh Generation Procedure
}

\author{
S. Cortez, J.C.P. Claro, J.L. Alves \\ Mechanical Engineering Department. University of Minho \\ CT2M - Centre for Mechanical and Materials Technologies \\ Guimarães, Portugal \\ \{scortez, jcpclaro, jlalves\}@ dem.uminho.pt
}

\begin{abstract}
The Finite Element (FE) Analysis is an essential tool to study the biomechanical behaviour of the spine, and particularly of the intervertebral disc (IVD). The 3D reconstruction of a patient-oriented IVD has numerous obstacles. Difficulties arise mainly due to the complexity and dimensions of the IVD's constituent structures. The lack of detailed data in conventional imaging techniques causes further problems in the IVD finite element mesh generation and analysis. The accuracy of the FE computation increases if the geometry of model resembles, for instance, the natural smoothness of real anatomical structure. Accounting to this, the main idea of this work is to evaluate the impact of the reconstruction parameters by comparing the final 3D geometrical reconstruction by finite elements with an initial well-defined geometry of a given anatomical structure, and to develop the procedures for the 3D mesh generation from 2D medical imaging.
\end{abstract}

Keywords-biomechanics; intervertebral disc; finite elements; medical imaging; resolution

\section{INTRODUCTION}

The finite element analysis has contributed to improve our understanding on the biomechanics of the spine, specially the IVD [1]. In the majority of the cases, biological structures are not easy to study in-vivo and only computational models and numerical simulations are easily accessible allowing an "inside" view. IVDs are no exception, as they are hardly in-vivo accessible. Virtual 3D models, such as 3D FE meshes, can be used to "virtualize" the internal structures of the Human body, and thus contributing to medical diagnosis of pathologies; to decide rehabilitation strategies; to virtualize surgeries and to an a priori evaluation of surgical strategies. However, the $3 \mathrm{D}$ reconstruction process of a patient-oriented data is somewhat complex and is known to have several problems causing numerous difficulties in the FE analysis, such as the identification of tiny or too complex geometric details. The accuracy of the FE computation increases if the geometry of model resembles the real structure.

In general, a FE model can be created from a solid voxel-based $3 \mathrm{D}$ model obtained from $3 \mathrm{D}$ reconstruction of a set of $2 \mathrm{D}$ medical images. Most of the 3D models proposed in literature are based on specific and real data, essentially computer tomography (CT), and significantly different geometries can be seen. Such big differences when dealing with similar real structures reveal the lack of robustness of the $3 \mathrm{D}$ reconstruction process, and not simply real differences on medical images and/or anatomical information and histological observations. However, it is also known that both the type of medical imaging technique and image resolution play a key role in the $3 \mathrm{D}$ reconstruction process.

Sometimes these geometrically-based models do not provide complete information about the different structures due to the low resolution of most medical images, i.e., very tiny details and tiny structures have a high probability of being lost "inside" the resolution. On the other hand, some soft tissues of the IVD, especially nucleus pulposus and annulus fibrosus, have similar densities and constitution, what makes 3D reconstruction even more difficult and imaging segmentation a very user-dependent task. CT images has proved to be a good tool to aid in the segmentation of bony structures but turned out to be less efficient than those obtained by MRI in the detection of soft-tissues such as the ones within the IVDs.

\section{CASE Study}

A three-dimensional voxel-based geometric model of a goat motion segment was created from MRI data. An imaging processing software (ScanIP/Simplewire) was used for imaging segmentation (identification of different structures and tissues), both in images with lower (normal MRI) and higher (micro-MRI) resolutions. Fig. 1 shows two different models based on the aforementioned medical images with two different resolutions.

Several segmentation algorithms were tested and applied on the goat motion segment (MS). However, none of these algorithms allowed to achieve an automatic segmentation of all the structures of the MS, especially regarding the intervertebral disc due to the low images resolution, lack of contrast between domains/structures and level of noise. Such difficulties made completely impossible to obtain a high level of confidence of the initial (real) geometry of the motion segment.

\footnotetext{
Master Thesis

Portuguese chapter of IEEE EMBS

$3^{\text {rd }}$ Portuguese Meeting in Bioengineering, February 2013

University of Minho
} 


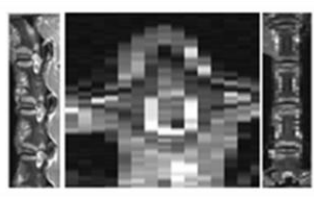

a)

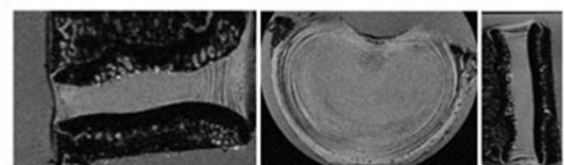

c)

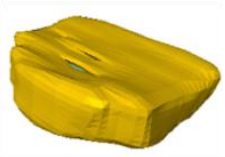

b)

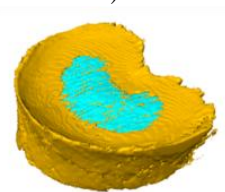

d)
Figure 1. The MRI (a) and micro-MRI (c) images of the lumbar goat IVD in different planes (sagittal, axial and coronal, respectively) and the $3 \mathrm{D}$ voxel-based geometric model ( $b$ and d) obtained after segmentation of each set of images. The first set of images consists of 14 sagittal, 336 axial and 83 coronal slices with $3 \mathrm{~mm}$ of thickness and $3.3 \mathrm{~mm}$ of slice spacing (voxel size of $0.3 \times 0.3 \times 3.3 \mathrm{~mm}^{3}$ ). The second set has 250 sagittal, 124 axial and 185 coronal slices with $0.12 \mathrm{~mm}$ of slice thickness and $0.12 \mathrm{~mm}$ of slice spacing (voxel size of $0.12 \times 0.12 \times 0.12 \mathrm{~mm}^{3}$ ).

Thus, a new procedure was implemented in order to allow the study of the influence of both medical imaging resolution and FE mesh generation procedure on the quality and geometric accuracy of the final 3D geometric model by finite elements.

\section{METHODOLOGY}

\section{A. Virtual Voxel-based Model}

A hexahedral FE mesh (Fig. 2) of a Human lumbar motion segment (L4 - L5), originally created by T.H. Smit (available on the web from the ISB Finite Element Repository ), was reworked [2] and used as a reference geometry to allow a comparison with the final FE meshes obtained from the newly proposed FE mesh generation procedure.

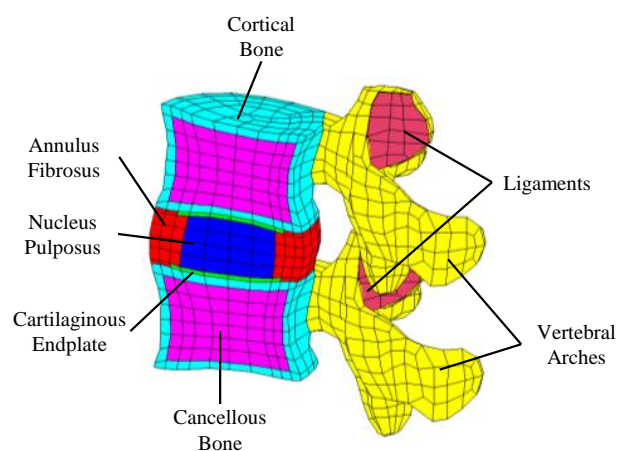

Figure 2. Hexahedral FE mesh of a Human lumbar motion segment.

The starting point for the generation of a virtual voxel-based segmented 3D geometrical model is the selection of a "real" geometry of a motion segment. Assuming this geometry (Fig. 2) as being representative of a real motion segment, a FORTRAN code was developed to virtually generate the segmented 2D medical images. Such 2D segmented images were then used to carry out the $3 \mathrm{D}$ reconstruction of the voxel-based geometric model, which will be used for the FE mesh generation procedure. Among others, this technique allows to study the influence of some data acquisition parameters, such as image resolution and slice thickness and spacing between slices; the 3D modelling virtualization was also used to study and understand the influence of image resolution on FE mesh generation procedures. The virtualization procedure runs as follows: firstly the global dimensions $(x, y, z)$ of the initial hexahedral FE mesh are determined. For this, the minimum and maximum coordinates are determined for each axis (Fig. 3a). The next step is to input the voxel size (dimensions in $X, Y$ and $Z$ ), which will drive the resolution specified for the $2 \mathrm{D}$ images. Given a $2 \mathrm{D}$ example, where a pixel corresponds to a voxel in $3 \mathrm{D}$, a mesh domain and pixel size are defined and, then, a first pixel is generated in the centre of domain (Figure 3b). Subsequently, pixels and voxels structure is created for the entire 3D domain (Fig. 3c,d), generating from the initial FE mesh a 3D matrix of "empty" voxels.
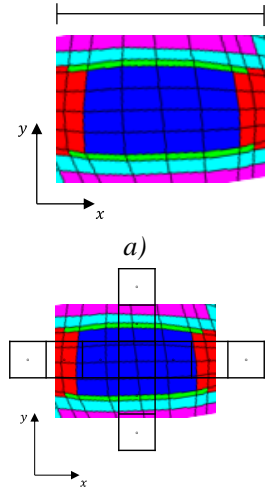

c)

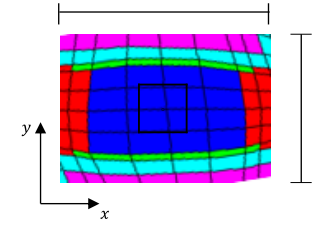

b)

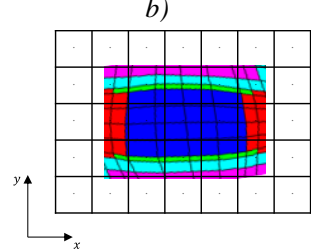

d)
Figure 3. A two-dimensional illustration of the (three-dimensional) creation of the grid of pixels (voxels).

\section{B. Voxel_ID searching algorithm}

Next step is to determine the segmentation data at each voxel. In order to identify the material ID to be assigned to each voxel, it is necessary to find the original finite element to which the centre of a given voxel belongs to. Once found, the material ID of the finite element is automatically assigned to the voxel, and so on for all voxels. At the end of this virtual imaging processing, a voxel-based 3D reconstructed geometrical model is obtained. Each FE mesh consists on the following data: nodes and their spatial coordinates, finite elements and their connectivity and material ID assigned to each finite element. Since the original FE mesh is a hexahedral mesh, each element is defined by eight nodes, and each node has a given spatial organization inside the finite element. For simplicity, each finite element is formulated in a canonical (or natural) frame, and then the equivalence between Cartesian and canonical frame is derived from interpolation functions (also known as shape functions) which will allow to design the mathematical procedure to identify within each finite element a given voxel is.

Being $\boldsymbol{x}$ the Cartesian coordinates of the central point of a given voxel, the mathematical problem can be defined as: find if point $\boldsymbol{x}$ is or is not inside of a given finite element $F E$ of the original FE mesh. The following nonlinear equation can be formulated, 


$$
\boldsymbol{x}-\boldsymbol{x}_{\mathrm{FE}}(\xi, \eta, \zeta)=\mathbf{0}
$$

where the unknowns are the 3 canonical coordinates $(\xi, \eta, \zeta)$.

However, since the relationship between $\boldsymbol{x}_{\mathrm{FE}}(\xi, \eta, \zeta)$ and $(\xi, \eta, \zeta)$ depends of the shape functions, it is necessary to use an iterative numerical method, such as the Newton-Raphson method. So, after having iteratively determined the solution of the abovementioned problem, one needs to decide if point $\boldsymbol{x}$ is or is not inside the finite element under analysis, i.e., point $\boldsymbol{x}$ is inside the finite element (FE) if and only if

$$
-1 \leq \xi \leq 1 \wedge-1 \leq \eta \leq 1 \wedge-1 \leq \zeta \leq 1
$$

Finally, after finding that a given voxel is inside of a given finite element, the next step is to identify the material ID of the element in order to assign the same ID to the voxel. A matrix of pixels is superposed on the real domain, and the 2D medical images cab be virtually generated and segmented slice-by-slice (Fig. 4). At the end of this procedure, the generated 3D voxel-based geometry will be used for the 3D FE mesh generation procedure.
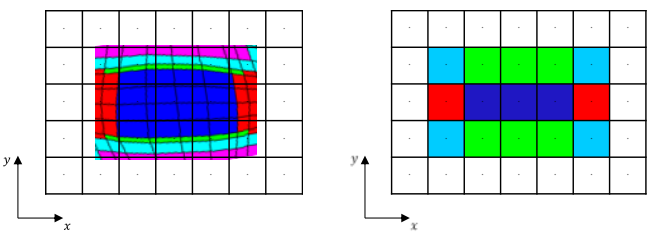

Figure 4. Scheme of the virtual segmentation on the pixels' matrix.

The voxel's dimensions are one of the most important parameters regarding the quality of the $3 \mathrm{D}$ geometrical reconstruction and later the representativeness and geometrical accuracy of generated 3D FE meshes. Voxel's dimensions are driven by the in-plane pixel's size and slice spacing, and shall depend on the geometry of anatomical regions under analysis. It is necessary to identify the tiniest geometrical detail to be taken into account, and thus to choose a voxel's dimension small enough to capture such geometrical details. In the case of a motion segment and, in particular, in case of the Human motion segment under analysis, the tiniest geometrical feature to be captured is the cartilaginous endplate $(1 \mathrm{~mm}$ of thickness). Hence, in this work two different voxel's dimensions were selected for analysis: $0.3 \times 0.3 \times 3.3$ and $0.12 \times 0.12 \times 0.12 \mathrm{~mm}^{3}$.

Using Matlab (Mathworks Inc., Natick, MA), a plane sectioning procedure was implemented in order to allow a visual analysis of the influence of image resolution on the $3 \mathrm{D}$ voxels' 3D geometrical reconstruction. Figures 4 and 5 show a comparison between two sections of either the initial geometrical domain or the 3D voxel-based models obtained from the virtual voxel-based model generation procedure previously described.

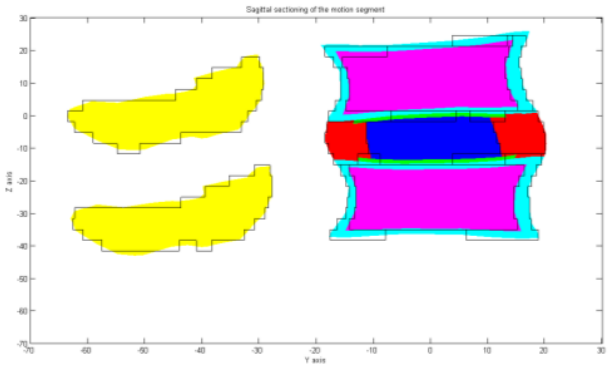

Figure 4 - Sagittal sectioning of the motion segment with a voxel size of $0.3 \times 0.3 \times 3.3 \mathrm{~mm}^{3}$. Coloured areas represent the initial geometry while dark lines represent simply the countours of the voxel-based 3D model.

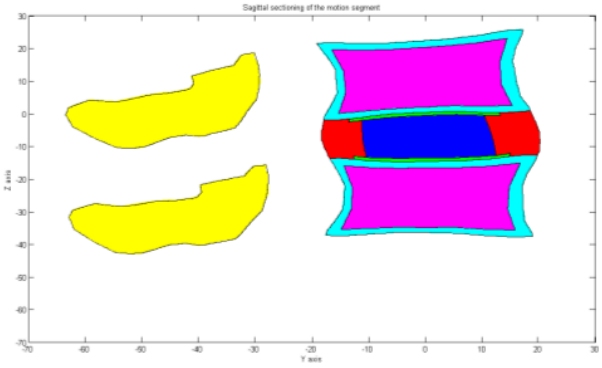

Figure 5. Sagittal sectioning of the motion segment with a voxel size of $0.12 \times 0.12 \times 0.12 \mathrm{~mm}^{3}$. Coloured areas represent the initial geometry while dark lines represent simply the countours of the voxel-based 3D model

Table I shows a comparison between global (motion segment and IVD) and local (each sub-domain of the motion segment) volumes, determined from both reference geometry and after segmentation for the two resolutions under analysis. The volumetric errors, in the case of the IVD for $0.3 \times 0.3 \times 3.3 \mathrm{~mm}^{3}$ voxel size, range between 3 and $7 \%$, while in case of the endplate is of $+113 \%$. This last strange result was found to be coupled with the elongated shape of the voxel. As previously mentioned, the voxel's dimension influences largely the outcome of the 3D model. In fact, in case of the voxel's size of $0.12 \times 0.12 \times 0.12 \mathrm{~mm}^{3}$ (Table II) the volumetric errors are practically null due to the similarity between the dimension of the voxel and the dimension of the anatomical regions to be discretized.

TABLE I. NUMBER OF VOXELS AND VOLUMES OF BOTH VOXEL AND INITIAL GEOMETRIES, FOR EITHER THE GLOBAL MOTION SEGMENT AND

\begin{tabular}{|c|c|c|c|c|c|}
\hline \multicolumn{6}{|c|}{ Voxel dimension $0.3 \times 0.3 \times 3.3 \mathrm{~mm}^{3}$} \\
\hline Nucleus & 25537 & 7584 & 8154 & 0.930 & $-7 \%$ \\
\hline Cortical Bone & 79369 & 23573 & 25149 & 0.937 & $-6 \%$ \\
\hline Cancellous bone & 118953 & 35329 & 34331 & 1.029 & $3 \%$ \\
\hline Vertebrae & 137105 & 40720 & 41069 & 0.992 & $-1 \%$ \\
\hline TOTAL IVD & 71707 & 21297 & 20384 & 1.045 & $4 \%$ \\
\hline
\end{tabular}
INTERVERTEBRAL DISC OR CONSTITUINT SUBSTRUCTURES, FOR A VOXEL SIZE OF 0.3X0.3X3.3 $\mathrm{MM}^{3}$. 
TABLE II. NUMBER OF VOXELS AND VOLUMES OF BOTH VOXEL AND INITIAL GEOMETRIES, FOR EITHER THE GLOBAL MOTION SEGMENT AND INTERVERTEBRAL DISC OR CONSTITUINT SUBSTRUCTURES, FOR A VOXEL SIZE OF $0.12 \times 0.12 \times 0.12 \mathrm{MM}^{3}$.

\begin{tabular}{|c|c|c|c|c|c|}
\hline \multicolumn{6}{|c|}{ Voxel dimension $0.12 \times 0.12 \times 0.12 \mathrm{~mm}^{3}$} \\
\hline Material & Number of Voxels & $\begin{array}{c}\text { Vv, Volume of voxel } \\
{\left[\mathrm{mm}^{3}\right]}\end{array}$ & $\begin{array}{c}\text { Vi Volume of initial geometry } \\
{\left[\mathrm{mm}^{3}\right]}\end{array}$ & $\begin{array}{l}\text { Ratio } \\
\text { Vv/Vi }\end{array}$ & Error \\
\hline Nucleus & 4721782 & 8159 & 8154 & 1.001 & $0 \%$ \\
\hline Annulus & 6179904 & 10679 & 10678 & 1.000 & $0 \%$ \\
\hline Endplate & 895486 & 1547 & 1552 & 0.997 & $0 \%$ \\
\hline Cortical Bone & 14563557 & 25166 & 25149 & 1.001 & $0 \%$ \\
\hline Cancellous bone & 19864602 & 34326 & 34331 & 1.000 & $0 \%$ \\
\hline Vertebrae & 23778971 & 41090 & 41069 & 1.001 & $0 \%$ \\
\hline Ligaments & 324258 & 560 & 560 & 1.000 & $0 \%$ \\
\hline TOTAL & 70328560 & 121528 & 121493 & 1.000 & $0 \%$ \\
\hline TOTAL IVD & 11797172 & 20386 & 20384 & 1.000 & $0 \%$ \\
\hline
\end{tabular}

\section{Mesh Generation Procedure}

After the 3D voxel-based geometrical reconstruction, next step is the application of a specific FE mesh generation procedure. Based on the work of Labelle and Shewchuk (2007) [3], a tetrahedral FE mesh was created keeping in mind the nature and smoothness of the boundaries of soft tissues. To use this grid-based algorithm, the first step is to superimpose a 3D grid structure (a body-centred lattice - BCC) over the 3D voxel-based model. An octree subdivision can be introduced in the neighbourhood of volume boundaries. The geometrical accuracy of the FE mesh depends on the grid size dimensions. Thus, the characteristic dimension of the isotropic grid shall be studied in detail in order to allow the generation of a "good quality" FE mesh. Throughout this study four grid sizes were used: $0.25,0.5,1$ and $2 \mathrm{~mm}$. The FE mesh generation procedure requires two input parameters. The first one is $\mathrm{N}$, which is associated with the definition of the grid size. Depending on the voxel's diagonal, it is possible to determine the value of $\mathrm{N}$ for each of the above mentioned grid sizes to be studied. Parameter $\mathrm{N}$ is a function of the required grid size and of the voxel diagonal $\mathrm{l}$ :

$$
\text { 'Grid Size' = N x }(l / \sqrt{ } 3)
$$

The second parameter required for the FE mesh generation is $\sigma$. This parameter defines the standard deviation of the Gaussian distribution used for sampling. In other words, while $\mathrm{N}$ defines the grid size and determines the size of the BCC lattice superposed to the voxelized data, $\sigma$ defines the amount of neighbouring data that shall be taken into account for sampling the voxel data into the vertices and centre point of the grid's BCC lattice, i.e., the determination of the material ID to be assigned to each vertex and centre of the square in the grid. The weight to be assigned to each data decreases with the distance in agreement with a Gaussian distribution, and the shape of Gaussian distribution depends on $\sigma$. When $\sigma$ is very high, the area to be searched increases (the cut-off radius is $2 \sigma$ ), what usually introduces an additional smoothing of boundaries, and consequently the loss of some anatomical geometrical features.

On the other hand, when the value of $\sigma$ is too small, the smoothing is less pronounced and, because the volume of data considered for sampling is small, sometimes the discrete voxel structure is wrongly captured. The values of these two parameters $(\mathrm{N}$ and $\sigma$ ) were carefully studied and defined in order to adapt the FE mesh generation procedure for the case of a motion segment like the one proposed in this work (Fig. 6).

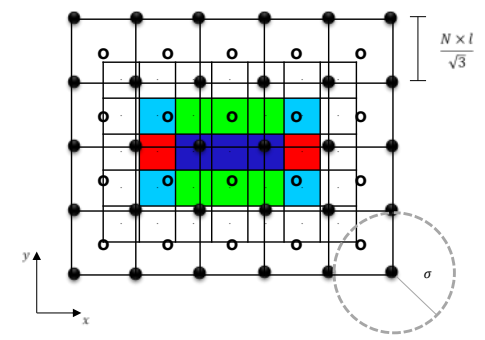

Figure 6. Scheme of the FE mesh generation parameters (grid size, $\mathrm{N}$ and $\sigma$ ).

After the definition of the input parameters, the tetrahedral FE meshes are generated based on an algorithm which is based on four steps: sampling at grid points, computation of cutting points, warping of some grid points and, finally, the application, at the grid cell level, of the pre-defined stencils (geometric arrangement of a nodal group that relate to the point of interest by using a numerical approximation) for the generation of a tetrahedral FE mesh [3]. These four steps are briefly described below:

Sampling at grid points - the scalar value for each vertex of the grid needs to be determined. Each vertex is associated with a mark: positive (inside), negative (outside), and zero (on the boundary). For interior grid cells, all vertices are labelled with ' + ' mark. In case of multi-material domains, to each vertex is assigned the material ID of the domain in which the vertex shall be considered. Sampling algorithm is based on a Gaussian distribution centred on the vertex.

Cutting points - the computation of a cutting point is required when, for a given edge of the $\mathrm{BCC}$ grid, one point is positive and the other is negative (or in case of different material IDs assigned to the two points of an edge). In these cases it is necessary to compute the cutting point where the edge crosses the zero-surface or a given domain boundary.

Warping of some grid points - In some cases it may happens that a cutting point can exist very close to one point of the grid. In these cases, when a cutting point is too close to a given vertex, one can assume that the cutting point violates the vertex and it is better to warp the grid by moving the vertex to the cutting point.

Divide grid cells - For each BCC cell, and after sampling, cutting points evaluation and warping, it is necessary to generate the FE mesh. The technique is simply to use some predefined stencils to fill-in each grid cell with tetrahedra, while respecting the outside and internal boundaries previously identified. The choice of the right stencil to be applied depends 
on the evaluation of signs and/or material IDs of the vertices of the grid cell under evaluation. Exemplifying in 2D, there are cases in which the four vertices and the central point of the grid may generate more than four triangles with different materials.

In order to understand the role of parameters $\mathrm{N}$ and $\sigma$ on the FE mesh generation, several values of these two parameters were selected. The rationale behind this choice is that value of parameter $\sigma$ must be coupled with grid size, and thus coupled to the value of N. Table III shows a summary of the global (full motion segment and IVD) and local (each structure or material) volumetric errors between the reference geometry and the generated FE mesh.

TABLE III. VOLUMES OF THE DIFFERENT MATERIALS OF THE FE MESHES GENERATED FROM A VOXEL SIZE OF $0.3 \times 0.3 \times 0.3$ AND $0.12 \times 0.12 \times 0.12 \mathrm{MM}^{3}$

\begin{tabular}{|c|c|c|c|c|}
\hline Voxel's Dimension $\left[\mathrm{mm}^{3}\right]$ & \multicolumn{2}{|c|}{$0.3 \times 0.3 \times 3.3$} & \multicolumn{2}{|c|}{$0.12 \times 0.12 \times 0.12$} \\
\hline Grid Dimension [mm] & \multirow{2}{*}{\multicolumn{2}{|c|}{0.26}} & \multirow{2}{*}{\multicolumn{2}{|c|}{4.17}} \\
\hline $\mathbf{N}$ & & & & \\
\hline$\sigma$ & 0.26 & 0.52 & 4.17 & 8.33 \\
\hline Number of Nodes[x10E6] & 2.3 & 2.3 & 2.2 & 2.2 \\
\hline $\begin{array}{c}\text { Number of FEs [x10E6] } \\
\text { Volume }\left[\mathrm{mm}^{3}\right] \text { (Error) }\end{array}$ & 12.8 & 12.7 & 12.0 & 12.1 \\
\hline Vertebrae & $41591(1 \%)$ & $42168(3 \%)$ & $41176(0 \%)$ & $41100(0 \%)$ \\
\hline Cortical Bone & $23953(-5 \%)$ & $24384(-3 \%)$ & $25314(1 \%)$ & $25373(1 \%)$ \\
\hline Cancellous Bone & $35905(5 \%)$ & $36698(7 \%)$ & $34404(0 \%)$ & $34313(0 \%)$ \\
\hline Ligaments & $549(-2 \%)$ & $389(-31 \%)$ & $536(-4 \%)$ & $390(-30 \%)$ \\
\hline Annulus Fibrosus & $10863(2 \%)$ & $10873(2 \%)$ & $1525(-2 \%)$ & $1458(-6 \%)$ \\
\hline Endplate & $3469(123 \%)$ & $3429(121 \%)$ & $10709(0 \%)$ & $10724(0 \%)$ \\
\hline Nucleus Pulposus & $7923(-3 \%)$ & $7915(-3 \%)$ & $8189(0 \%)$ & $8186(0 \%)$ \\
\hline IVD & $22256(9 \%)$ & $22217(9 \%)$ & $20423(0 \%)$ & $20368(0 \%)$ \\
\hline Total & $124254(2 \%)$ & $125856(4 \%)$ & $121853(0 \%)$ & $121543(0 \%)$ \\
\hline
\end{tabular}

It is obvious that, for the case of a voxel size of $0.3 \times 0.3 \times 3.3$ $\mathrm{mm} 3$, the volumetric errors are higher than those for the other cases, and are more relevant for the case of the endplate. This happens because large part of this structure is eliminated, as it can be seen in Fig.8a. For the voxel size of $0.12 \times 0.12 \times 0.12$ $\mathrm{mm}^{3}$, the generated FE meshes have an IVD's volumetric error close to zero. Throughout the FE mesh generation process, it was possible to conclude that a grid size of $0.5 \mathrm{~mm}$ was the best compromise between the FE mesh generation computational effort and global geometrical accuracy. In more details it could be confirmed that with a grid size of $0.5 \mathrm{~mm}$, even the smallest structure - like the endplate - was geometrically correctly and accurately described by the FE meshes. Therefore, it can be stated that the choice of the grid's size depends essentially on the size of the smallest structure/region that shall be geometrically captured by the FE mesh. As parameter $\mathrm{N}$ defines the grid size as a function of the diagonal of the voxel, it was found that in some cases the voxel size was not compatible with the FE mesh generation software (usually in the case of very low values of $\mathrm{N}$ ).

Using the software GID ${ }^{\circledR}$, a FEA pre- and post- processor, the external boundary of the obtained FE meshes can be plotted for the two different resolutions. After the analysis of all the results obtained during the $\mathrm{FE}$ mesh generation procedure, the FE meshes obtained with the grid size of $0.5 \mathrm{~mm}$ and the higher $\sigma$ were chosen as the best ones. Figure 8 shows these FE meshes for resolutions of $0.3 \times 0.3 \times 3.3$ and $0.12 \times 0.12 \times 0.12$ $\mathrm{mm}^{3}$, with a grid size of $0.5 \mathrm{~mm}$ and $\sigma$ of 0.52 and 8.33, respectively. By comparing the two different meshes, there were no improvements in the final FE mesh obtained with a voxel size of $0.3 \times 0.3 \times 3.3 \mathrm{~mm}^{3}$, where the geometry with a "stairs' effect" is present (Fig. 7a). However, a better resolution of the voxel-based geometry always allows better results of the geometric definition of the anatomical structures by a FE mesh (Fig. 7b).

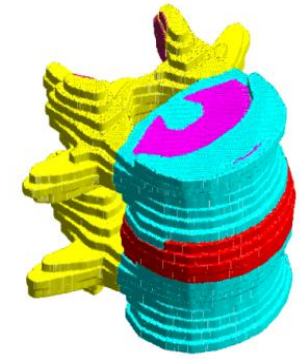

a)

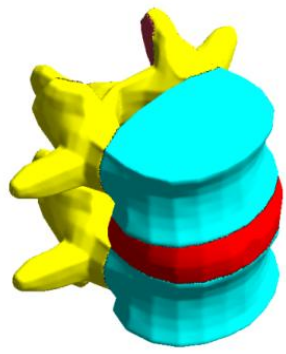

b)

Figure 7. FE meshes obtained for voxel's dimension of a) $0.3 \times 0.3 \times 3.3$ and b) $0.12 \times 0.12 \times 0.12 \mathrm{~mm}^{3}$ with the grid size of 0.5 and the largest value of $\sigma$.

\section{FE Mesh Simplification Procedure}

A FE mesh obtained by the above process is usual to be computationally very expensive. This implies the need of decreasing its size, applying a simplification procedure, although always keeping in mind its geometry and quality. The FE mesh simplification process consists in reducing the number of tetrahedra in the FE mesh while keeping the shape, volume and boundaries between different structures. In this method, based on a cost function, local simplification operators are iteratively applied, which contract the edges of the elements taking into account the quality and geometric error of both boundaries and FEs. The optimum location of the new node depends primarily on the dihedral angles formed by adjacent elements when the edge is contracted (avoiding/eliminating dihedral angles close to $0^{\circ}$ or $180^{\circ}$ ).

A simplification procedure was studied and applied to the FE meshes previously generated with a voxel's dimension of $0.12 \times 0.12 \times 0.12 \mathrm{~mm}^{3}$. This simplification algorithm implies the input of a set of parameters to control the simplification process. However, in this study only two of these parameters are used: one to control the scaled Jacobian (-t0.05), defined to assure a minimum quality of the simplified tetrahedra, while the second parameter is simply the desired number of tetrahedra (to stop the simplification process). At the end, a much coarser FE mesh is obtained with a similar finite element density at both vertebrae and IVD. However, our paramount goal is to generate a good quality FE mesh of the IVD, creating smaller FE in the IVD region and larger FE in other structures of the motion segment. For that, a sizing field, defining a FE mesh refinement gradient, can be defined and applied.

The sizing field, which can be absolute (-s) or relative (-r), is the maximum edge length allowed at a given spatial point. In this study, the usage of these two options was evaluated. After the FE mesh generation (with a voxel's size of $0.12 \times 0.12 \times 0.12$ $\mathrm{mm}^{3}$ ), the file was converted to a VTK format file and two additional sizing fields added. The values specified by the user for the edge sizing field (defined at each node) inside and outside the IVD region were 1 and $10 \mathrm{~mm}$, respectively. Fig. 8 show the FE mesh with an absolute sizing field applied and the average edge size in the motion segment. The same results for the relative sizing field are shown in Fig. 9. 


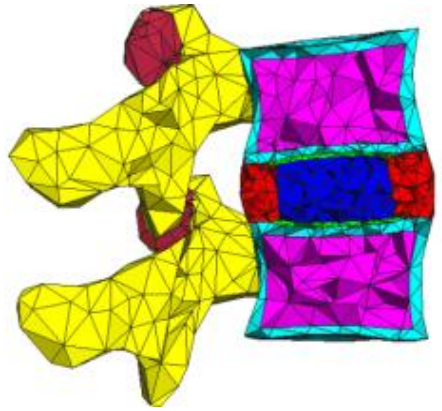

a)

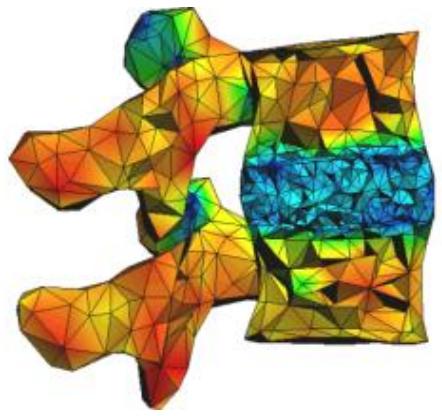

b)

Figure 8. FE mesh obtained by simplification algorithm with an absolute sizing field applied.

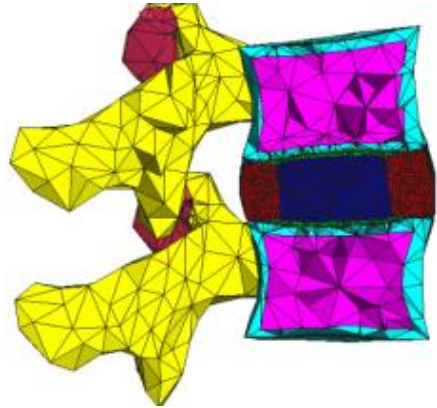

a)

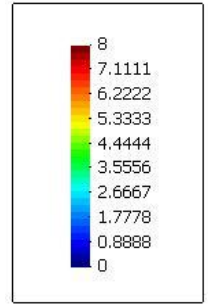

It is possible to see (Fig. 8 and Fig. 9) that there is a noticeable difference in the overall elements size between the IVD and the other regions of the motion segment. A very refined mesh in the IVD can be seen, simultaneously with a very coarse one on the remaining structures of the motion segment. Furthermore, this simplification procedure allowed to generate FE meshes with a visible geometrical accuracy, being computationally much more effective than the former ones.

\section{CONCLUSION}

This new algorithm allowed to obtain virtually a set of 2D segmented medical images, and to reconstruct a voxel-based 3D model with a user-defined resolution (or voxel's dimensions, i.e., in-plane pixel size and slice spacing). The two different voxel sizes studied allowed to conclude that the resolution of the medical images influences mostly the geometrical accuracy of the reconstructed 3D models. Images with higher resolutions allowed building models with higher geometric accuracy. The FE mesh simplification procedure also allowed demonstrating the possibility to completely master the simplification process by defining a non-uniform and user-definable sizing field. For instance, the definition of a higher FE mesh refinement in regions of interest (like the IVD) can be easily obtained using the proposed FE mesh simplification algorithm. Essentially, an absolute and a relative sizing field allow to manipulate edges' sizes, and thus FE sizes, in different regions/structures of the global FE mesh. It is a powerful tool to decrease the size (number of nodes and number of elements) of the FE meshes, simultaneously keeping the geometric accuracy and FE mesh quality. Finally, this work allowed to conclude that following the proposed algorithm it is possible to describe, with a reasonable geometrical accuracy and a good quality FE mesh, the bio-structures of a motion segment in order to develop computational studies by finite element of the biomechanical behaviour of the Human spine.

\section{ACKNOWLEDGMENT}

The authors gratefully acknowledge the support of the European Project: NP Mimetic - Biomimetic Nano-Fiber Based Nucleus Pulposus Regeneration for the Treatment of Degenerative Disc Disease, funded by the European Commission under FP7 (grant NMP3-SL-2010-246351).

\section{REFERENCES}

[1] Schroeder, Y., Huyghe J. M., van Donkelaar, C.C., Ito, K., A biochemical/biophysical 3D FE intervertebral disc model, Biomechanics and Modeling in Mechanobiology, 2010, Vol. 9, 641-650

[2] Castro, A., Wilson, W., Huyghe, J. M., Ito, K., Alves, J.L., A Poroelastic approach for an open source finite element model of the intervertebral disc, Proceedings of CMBBE2012 Symposium, 2012

[3] Labelle, F. and Shewchuk, J. R., Isosurface stuffing: Fast tetrahedral meshes with good dihedral angles, ACM Transactions on Graphics (TOG), 2007, Vol. 26(3), 57.1-57.10 